\title{
Solitons in a medium with linear dissipation and localized gain
}

\author{
Dmitry A. Zezyulin, ${ }^{1, *}$ Yaroslav V. Kartashov, ${ }^{2}$ and Vladimir V. Konotop ${ }^{1}$ \\ ${ }^{1}$ Centro de Física Teórica e Computacional, and Departamento de Física, Faculdade de Ciências, \\ Universidade de Lisboa, Avenida Professor Gama Pinto 2, Lisboa 1649-003, Portugal \\ ${ }^{2}$ ICFO-Institut de Ciencies Fotoniques, and Universitat Politecnica de Catalunya, \\ Mediterranean Technology Park, 08860 Castelldefels (Barcelona), Spain
}

(Dated: May 28, 2022)

\begin{abstract}
We present a variety of dissipative solitons and breathing modes in a medium with localized gain and homogeneous linear dissipation. The system posses a number of unusual properties, like exponentially localized modes in both focusing and defocusing media, existence of modes in focusing media at negative propagation constant values, simultaneous existence of stable symmetric and anti-symmetric localized modes when the gain landscape possesses two local maxima, as well as the existence of stable breathing solutions.
\end{abstract}

PACS numbers: $42.65 . \mathrm{Tg}, 42.65 . \mathrm{Sf}$

Dissipative solitons appear in a variety of forms and settings. They form in laser systems with saturable gain and absorption [1], in homogeneous materials where light evolution can be described by the cubic-quintic Ginzburg-Landau (GL) equation [2], and in systems with spatially localized gain and uniform nonlinear losses $[3-$ 8]. A characteristic feature of all these systems is that the existence of dissipative solitons there is mediated by the competition of gain and losses having different physical origin. Thus, in the cubic-quintic GL equation solitons exist because of exact balance of the linear losses, cubic gain, and quintic losses. Likewise, solitons can also form because cubic losses compensate the spatially localized gain. This has been established in one-dimensional settings in the presence of a linear localized potential [3] or lattice [4], in two-dimensional problems in planar waveguide arrays [5], or lattices [6], or even in two-dimensional systems without additional refractive index modulations [7].

It is therefore important to elucidate simpler physical settings, where only the linear gain and losses result in formation of dissipative solitons. This is the goal of this Letter. More specifically, we report on the existence of stable dissipative solitons supported by a localized gain in a homogeneous Kerr medium where only the linear losses are present far from the center of gain landscape. This simple system possesses a number of interesting properties among which is the existence of localized modes even for negative propagation constant values, simultaneous stability of symmetric and antisymmetric modes in two-humped gain landscapes, as well as the existence of breathing modes.

We consider the nonlinear Schrödinger (NLS) equation

$$
i q_{z}=-q_{x x}-i\left[\gamma_{0}-\gamma(x)\right] q+\sigma|q|^{2} q .
$$

where $\sigma=1(\sigma=-1)$ corresponds to defocusing (focusing) nonlinearity, $\gamma(x)$ describes a localized gain concentrated in the domain (or domains) having a characteristic width $d$. Without loss of generality we rescale the back- ground dissipation to the value: $\gamma_{0}=1$, and consider the situation where $\max \gamma(x)>1$.

We look for spatially localized solutions obeying $\lim _{|x| \rightarrow \infty}|q(z, x)|=0$ and start with the stationary modes $q(z, x)=e^{i b z} w(x)$ where $w(x)=e^{i \theta(x)} u(x)$ with $u(x) \geq 0$ and $\theta(x)$ being real functions. Then, introducing the current density $j(x) \equiv \theta_{x} u^{2}$ one obtains from (1) the system describing stationary modes

$$
u_{x x}-b u+\sigma u^{3}-\frac{j^{2}}{u^{3}}=0, \quad j_{x}+[1-\gamma(x)] u^{2}=0
$$

In order to establish the domain of possible variation of the propagation constant $b$, we now address the limit $x \rightarrow \infty$ focusing on the exponentially decaying solutions, i.e. assuming $u \sim C e^{-\mu x}$, where $C$ and $\mu$ are positive constants. From the second of Eqs. (2) it readily follows that in this limit $j \sim C^{2} e^{-2 \mu x} / 2 \mu$. This suggests that in the first of equations (2) the term $\sigma u^{3}$ can be neglected in the limit $x \rightarrow \infty$, while substituting the asymptotic values of $u$ and $j$ in the terms which are left we obtain $b=\mu^{2}-1 /\left(4 \mu^{2}\right)$. It follows from this formula that the propagation constant for localized solutions can be either positive or negative even if the nonlinearity is focusing (contrary to what happens in uniform conservative systems or in dissipative systems [3, 4, [4, 6]). If $b \rightarrow+\infty$ then $\mu \rightarrow+\infty$ while if $b \rightarrow-\infty$ then $\mu \rightarrow+0$. Respectively, there must exist localized modes with $\mu>0$ even at $b=0$ while the spatial delocalization occurs in the limit $b \rightarrow-\infty$.

The condition of balance between the dissipation and gain (for $\gamma_{0}=1$ ) has the form $U=\int \gamma(x)|u|^{2} d x$ where $U=\int|u|^{2} d x$ is the energy flow. From the balance between the nonlinearity and diffraction we obtain

$$
b U=-\int\left|w_{x}\right|^{2} d x-\sigma \int|w|^{4} d x
$$

From this formula one readily concludes that for zero propagation constant, $b=0$, the mode should decay with $\mu=2^{-1 / 2}$, i.e. $\left|w_{x}\right|^{2} \not \equiv 0$, and hence (3) can be satisfied 
only in the focusing medium (i.e. at $\sigma=-1$ ). Thus the linear limit of the problem (i.e. $U \rightarrow 0$ ) corresponds to a localized mode with $b=b^{(0)}<0$ (indeed, in this case $\int|w|^{4} d x / U \leq \max |w|^{2} \rightarrow 0$ and can be neglected in (3i)). In the defocusing medium exponentially localized solutions exist only if $b<b^{(0)}$ while in the focusing medium dissipative solitons can exist with both positive and negative propagation constants, i.e. at $b>b^{(0)}$.

We start with the case of the gain localized in one spatial domain, setting $\gamma(x)=a e^{-x^{2} / d^{2}},(a>1$ characterizes the gain strength). By fixing the width of the gain landscape $d$ and changing $a$ we obtain the dependencies $b(a)$ and $U(a)$, which are depicted in Fig. 1 for $d=1$. These dependencies bifurcate from the linear limit corresponding to $U=0$ and to $b^{(0)} \approx-0.61$. The very fact of existence of localized linear modes with $b=b^{(0)}$ in inhomogeneous gain landscapes is known as the gainguiding effect (see e.g. [9]). In the linear case the guiding occurs for a particular value of $a=a^{(0)} \approx 1.98$. Focusing nonlinearity diminishes gain at which localization occurs, while in a defocusing medium a higher gain is required for localization (Fig. 1). In Fig. 1] we also show an example of a nonlinear mode. Increase of the gain results in decrease of $U$ in the focusing medium, that is also accompanied by expansion of the soliton. In the defocusing medium the growth of $a$ results in increase of the energy flow and progressive expansion of the soliton outwards the gain domain. Solitons are exponentially localized in the both focusing and defocusing media, as predicted by the analysis of (2) which showed that the current results in effective renormalization of the propagation constant. Physical understanding of the exponential localization in the defocusing medium, comes also form the observation that the current results in the effective self-focusing of the beam, what stems form the negative sign in front of $j^{2} / u^{3}$.

Linear stability analysis (confirmed by the direct propagation) indicates that the solitons in the focusing medium are unstable [see Fig. 2(a)], while in defocusing medium they are attractors and can be excited with a variety of inputs ranging from noisy to localized patterns. This difference in the stability can be understood from Eqs. (2). The solution represents a flow outwards the "source" (i.e. the gain domain). The defocusing nonlinearity enhances the outflow from the high intensity region thus contributing to the stability, while the focusing medium enhances the field concentration in the gain domain, what stimulates further growth of the peak amplitude.

Next we study the case of the gain landscape with two maxima at $x= \pm x_{0}$ considering $\gamma(x)=a e^{-\left(x-x_{0}\right)^{2} / d^{2}}+$ $a e^{-\left(x+x_{0}\right)^{2} / d^{2}}$, where $a>1$. Here one also can obtain modes both for focusing and defocusing media. In Fig. 3 we present symmetric (even) and antisymmetric (odd) modes. Both types of solutions bifurcate from the linear limit. The values of parameters $a$ and $b$ at which bifurca-
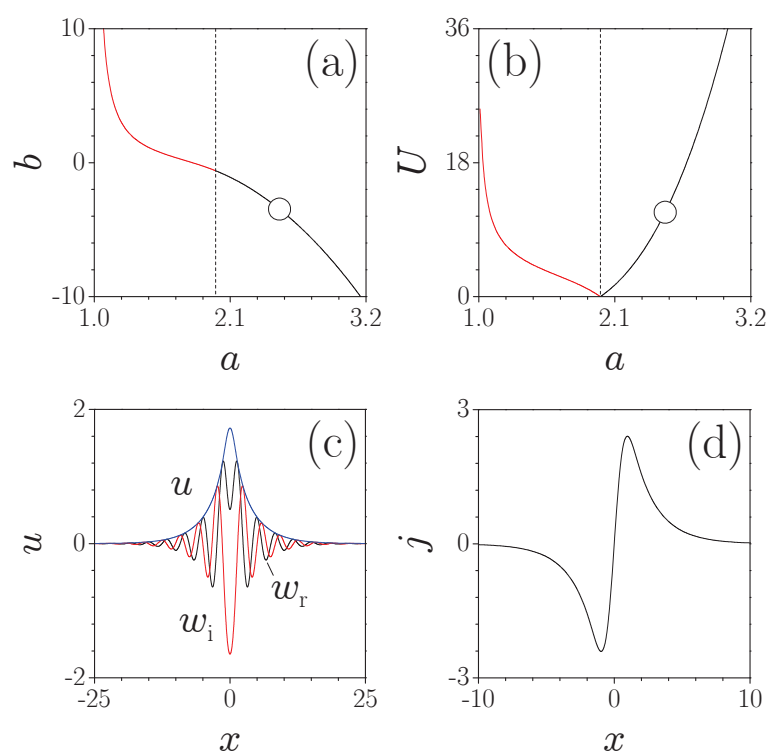

FIG. 1: Propagation constant (a) and energy flow (b) vs a for solitons supported by single gain channel. Red and black curves correspond to focusing and defocusing media. Dashed line indicates $a$ value corresponding to the linear limit. Profile of soliton (c) and current density (d) in defocusing medium at $a=2.5$ corresponding to the circles in (a) and (b).
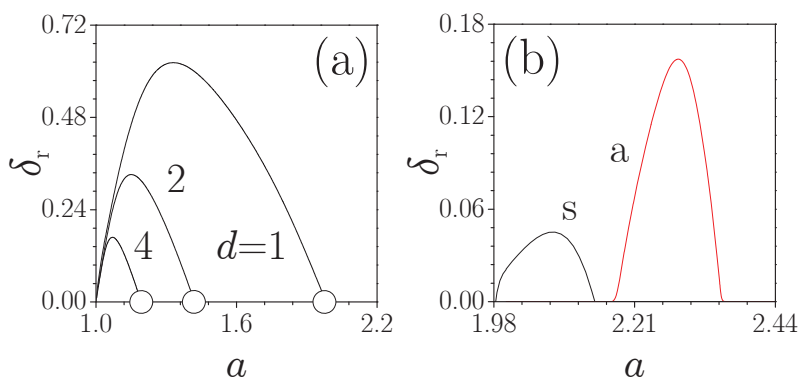

FIG. 2: (a) Instability increment $\delta_{r}$ vs a for solitons in a single gain channel in focusing medium. Circles indicate points beyond which solitons exist only in defocusing medium where they are stable. (b) $\delta_{r}(a)$ for symmetric "s" and antisymmetric "a" solitons in two gain channels with $d=1$ in defocusing medium.

tions take place are very close $\left(b_{s}^{(0)} \approx-0.61, a_{s}^{(0)} \approx 1.99\right.$ for symmetric, and $b_{a}^{(0)} \approx-0.60, a_{a}^{(0)} \approx 1.98$ for antisymmetric solitons). Closely to the linear limit and in the focusing medium the $U(a)$ curves for the symmetric and antisymmetric solitons are almost indistinguishable, while in the defocusing medium their energy flows may differ considerably [Figs. 3 (a) and (b)]. The examples of modes are shown in Figs. 3 (c) and (d).

Linear stability analysis reveals multiple alternating stability and instability domains (in $a$ ) for both symmetric and antisymmetric modes [Fig. 2 (b)]. A remarkable fact is that stable symmetric and antisymmetric modes co-exist at the same parameters of the system. Moreover, 

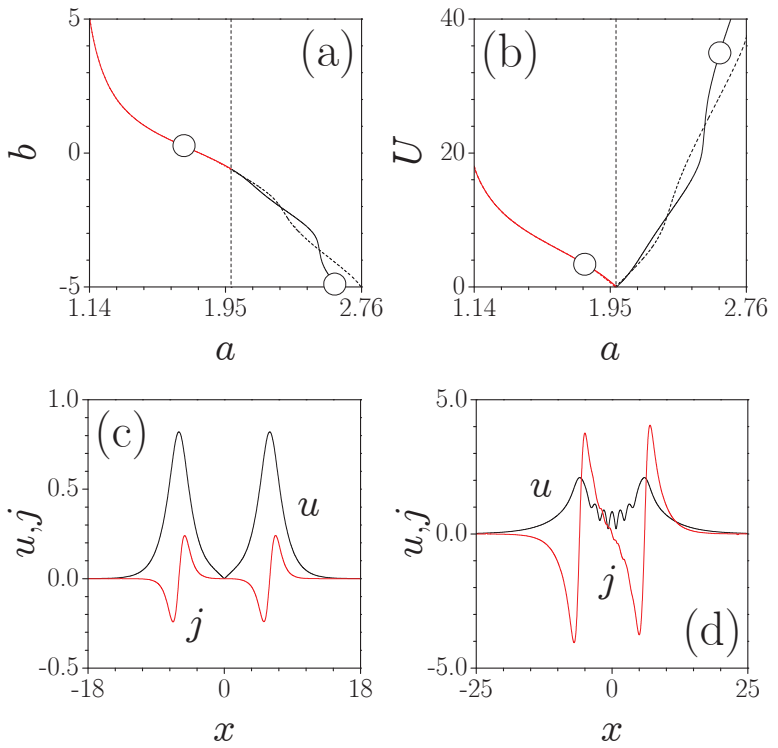

FIG. 3: Propagation constant (a) and energy flow (b) vs a for solitons supported by the two gain channels at $x_{0}=6$. Solid and dashed lines correspond to the symmetric and antisymmetric modes. Red and black lines correspond to the focusing and defocusing media. The circles correspond to the solitons shown in (c),(d). (c) Unstable antisymmetric soliton in the focusing medium at $a=1.8$. (d) The stable symmetric soliton in defocusing medium at $a=2.6$.

for the same value of $a$ both symmetric and antisymmetric modes in the defocusing medium can be attractors.

We found that for certain values of $a$ even input beams evolve into the symmetric stationary mode while odd input beams evolve into the antisymmetric mode, see panels (a) and (b) of Fig. 4 When one of the modes is unstable it usually evolves into a stable mode or into pulsating mode. An example of such behavior is shown in

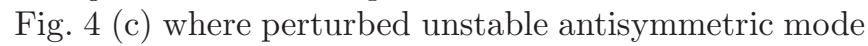
transforms into the breather. (We show only the initial stage of the evolution; simulations were performed up to $z=2 \cdot 10^{3}$ ). Dynamics shown in Fig. 4(c) is also illustrated in Fig. 5 where a corresponding trajectory in the phase space and dependence $U(z)$ are plotted.

Finally we explore the possibility of switching between stable solutions by changing the control parameter $a$. An example is shown in Fig. 团(d) where the input beam corresponds to the stable symmetric mode at $a=2.186$. At $z=100$ the strength of the gain $a$ is switched from 2.186 to 2.5 . Since the symmetric mode at $a=2.5$ is unstable, the switching results in the beam evolution into the stable antisymmetric mode corresponding to $a=2.5$.

To conclude, we found a variety of unusual properties of the solitons described by model (1), such as existence of exponentially localized modes with negative propagation constant values in both focusing and defocusing media, simultaneous stability of symmetric and antisym-
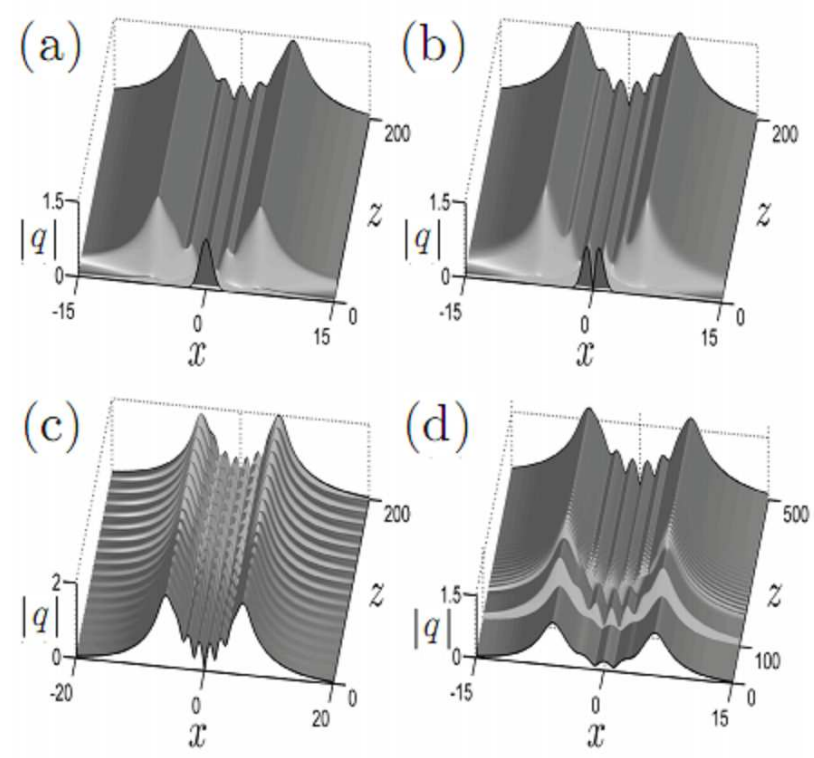

FIG. 4: Excitation of stable symmetric (a) and antisymmetric (b) solitons by symmetric and antisymmetric input beams in a system with two gain channels for $a=2.391$. (c) Excitation of a breather at $a=2.61$ starting with the unstable antisymmetric soliton. (d) Switching between stable symmetric and antisymmetric solitons stimulated by abrupt change of $a$ at $z=100$.
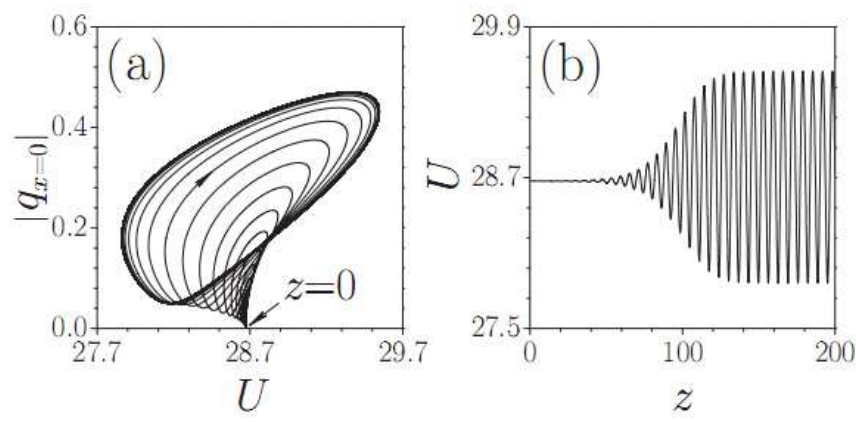

FIG. 5: The trajectory (panel a) and dependence $U(z)$ (panel b) corresponding to the dynamics shown in Fig. 4(c). The bold line shows the limit cycle.

metric solitons, and the existence of persistent breathers.

DAZ and VVK were supported by the grants SFRH/BPD/64835/2009 and PTDC/FIS/112624/2009.

[1] N. N. Rosanov, "Spatial hysteresis and optical patterns", (Springer, Berlin, 2010).

[2] N. Akhmediev and A. Ankiewicz, eds., "Dissipative Solitons: From Optics to Biology and Medicine", (Springer, Berlin, 2008)

[3] C.-K. Lam, B. A. Malomed, K. W. Chow, and P. K. A. Wai, Eur. Phys. J. - Special Topics 173, 233 (2009).

[4] Y. V. Kartashov, V. V. Konotop, V. A. Vysloukh, and L. 
Torner, Opt. Lett. 35, 1638 (2010)

[5] M. O. Williams, C. W. McGrath, and J. N. Kutz, Opt. Express, 11671, 18 (2010)

[6] Y. V. Kartashov, V. V. Konotop, V. A. Vysloukh, and L. Torner Opt. Lett. 353177 (2010)

[7] Y. V. Kartashov, V. V. Konotop, and V. A. Vysloukh,
Opt. Lett. 36, 82 (2011)

[8] C. H. Tsang, B. A. Malomed, C. K. Lam, and K. W. Chow Eur. Phys. J. D 59, 81 (2010)

[9] A. E. Siegman, J. Opt. Soc. Am. A 20, 1617 (2003) 